\title{
Nanotopograpical Analysis of Iron by AFM
}

\author{
NP Dahal and SK Lamichhane \\ Tribhuwan University, Department of Physics, Prithvi Narayan Campus, Pokhara \\ the_npd1@yahoo.com
}

\begin{abstract}
The roughness of the surface is sensed by the cantilever deflection of AFM, more the roughness of the surface greater will be the deflection and vice versa. The roughness of iron obtained from the AFM is depending upon the sanning area. The roughness of the surface can be studied from the analysis of the height profile and nano topography of the etched iron by AFM.
\end{abstract}

Key words: - Etching, AFM, Topography, Probe,

\section{Introduction}

Many fundamental studies in recent few years have dealt about the structure of iron. The surface energy, topographical study and the adhesion force between the iron molecules has made a revolution in the physics of conductor. Iron is a transition metal [1]. The transition metals are typical metals in that they tend to be bright, shiny, silvery solids. They all tend to conduct heat and electricity well. As iron is common and well known to all the people it is interesting and also important to study the topography in nanoscale. Iron atoms arrange themselves regularly in one crystal, and the direction of the arrangement of atoms differs among grains. The diameter of an iron atom is 0.25 nanometers; while that of a grain is usually 10 to $20 \mu \mathrm{m}$.

Iron atoms arrange themselves in one of two stable crystal structures called the body-centered cubic structure and the facecentered cubic structure [2]. Surface roughness is the one of the important physical properties of the metals like iron. Pure iron is known to be a soft metal, with a silver white or grayish color. One of the prime physical properties of Iron, which has contributed to its wide usage, is its properties of ductility, malleability and thermal conductivity.

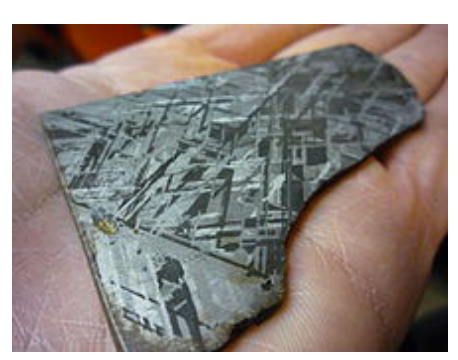

(a)

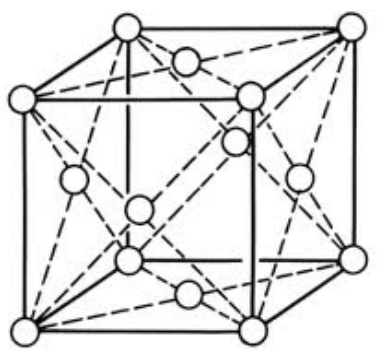

(b)

Fig 1: (a) Iron meteorites as a sixth most abundant element in the universe and (b) lattice structure of iron

Surface roughness and physical properties plays a critical role in adhesion. As the surface roughness approaches nano scale dimension, the surface roughness and area of contact should reach comparable dimensions such that apparent and true area of contact becomes approximately equal. In this regard, AFM nano adhesion experiment is increasingly popular to understand micro contact system. One of the major tools to study the image of iron is Atomic Force Microscope.

Atomic Force Microscope is a device designed to investigate both conductors and insulators on an atomic scale. Also Atomic force microscope (AFM) is a very high-resolution type of scanning probe microscopy, with demonstrated resolution on the order of fractions of a nanometer, more than 1000 times better than the optical diffraction limit [3]. The AFM is one of the foremost tools for imaging, measuring, and manipulating matter at the nanoscale. The AFM provides a three-dimensional surface profile and samples 
viewed by AFM do not require any special treatments.

\section{Methodology}

The entire system consisting of AFM head, optics, scanner (i.e. body of the AFM) is placed on the special table. Also the AFM consists of a cantilever with a sharp tip (probe) at its end that is used to scan the specimen surface [4]. The cantilever is typically silicon or silicon nitride with a tip radius of curvature on the order of nanometers. When the tip is brought into proximity of a sample surface, forces between the tip and the sample lead to a deflection of the cantilever. The AFM head includes a fine approach mechanism such that a motor capable of bringing down the tip coaxially to the scanner almost to the range where atomic forces of the sample atoms starts to operate. Once the tip is in suitable range, a scanner transducer controls the vertical and lateral position of the tip over the sample. The approach mechanism can be facilitating with the help of optics and a colored TV monitor. Sample movement, view of the cantilever with laser light can be observed during experiment on the TV screen is shown below.

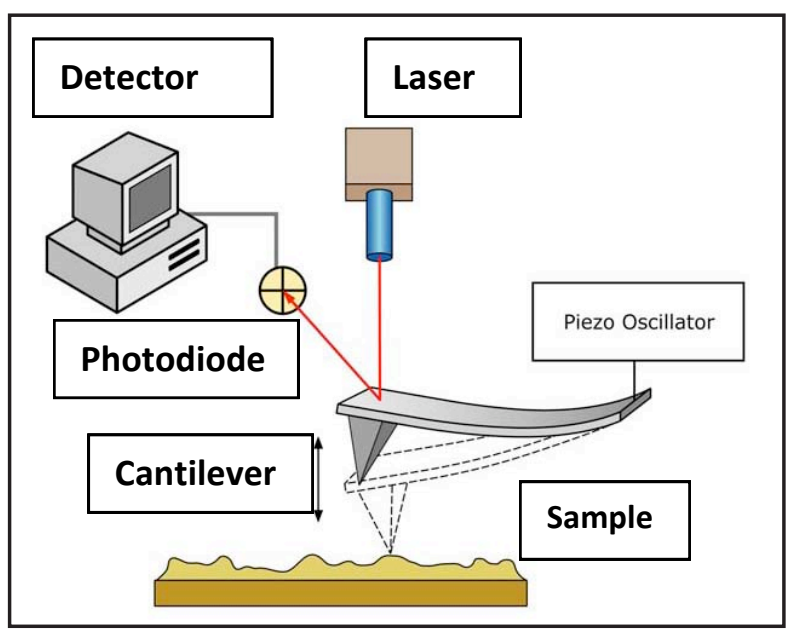

Fig 2: The schematic diagram of placement of sample on AFM

The topography of etched iron is taken in static mode, in which the cantilever is dragged across the surface of the sample and the contours of the surface are measured directly using the deflection of the cantilever [5]. Etching of iron is an integral part of micromachining. Etching of iron is strongly depends on crystal planes and hence is anisotropic. In this case a feedback mechanism is employed to adjust the tip-to-iron sample distance to maintain a constant force between the tip and the sample [6].

\section{Result and Discussion}

AFM has proved to be an important surface analysis technique with the capability of three-dimensional surface topography with sub nanometer and angstrom i.e., atomic resolution. The topographic information is obtained by scanning the surface with the sharp tip on a cantilever. During scanning two-dimensional plots of the feedback signal are used to develop a topographic image (map of surface structure) of the sample [7]. Because of the output signals of the feedback circuit is transformed to topography. The figure below shows the topography of etched iron as imaged by the AFM technique.

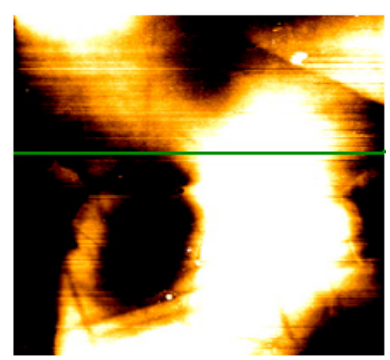

(a)

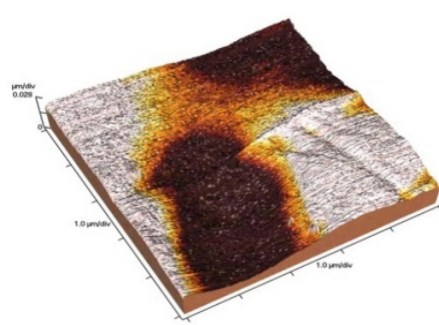

(b)
Fig 3: Iron Surface as observed by AFM (a) 2D: (10 (b) $3 D:(10 \mu \mathrm{m} \times 10 \mu \mathrm{m})$

Once the topographic data is generated from AFM scan the mean topographic roughness of an image is the arithmetic average of the absolute values of the surface height deviations measured from the mean [8]. Further $\mathrm{R}_{\mathrm{rms}}$ roughness is the root mean square average of height deviations taken from the mean and is calculated by

$$
R_{r m s}=\sqrt{\frac{\sum_{i=1}^{n}\left(Z_{i}-\bar{Z}\right)^{2}}{n}}
$$

Where, $Z_{i}$ is the image height and $n$ is the number of data points.

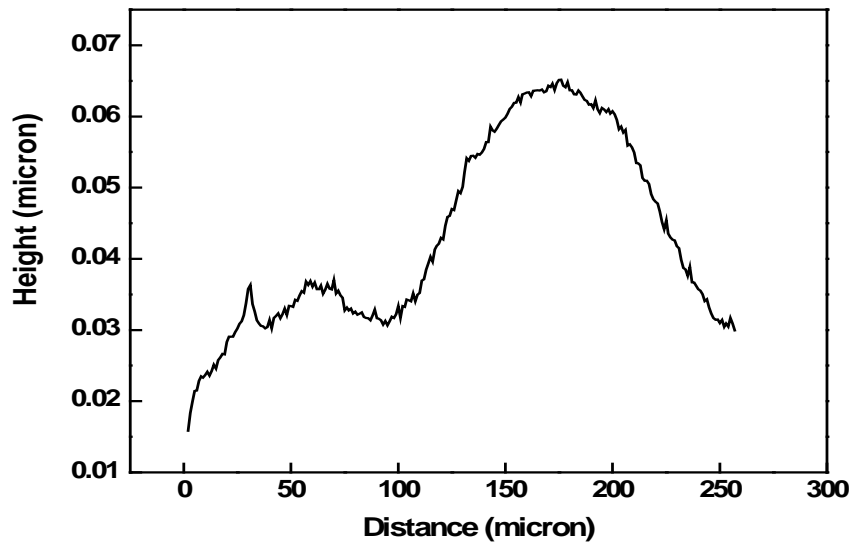




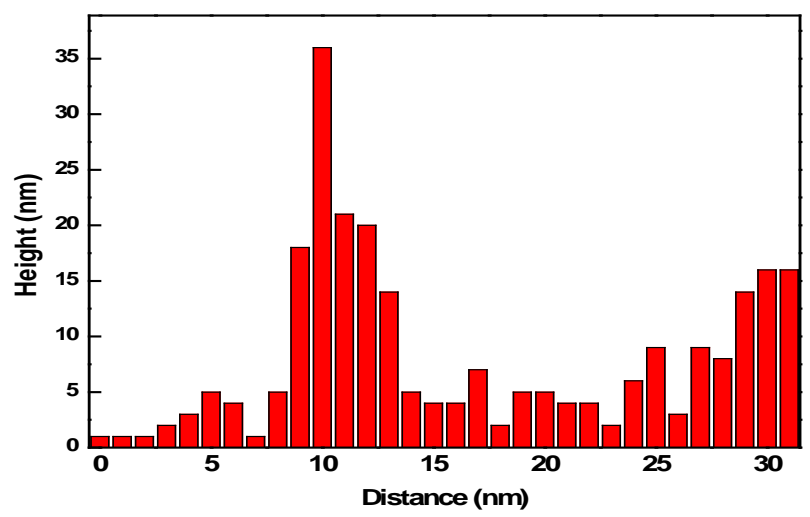

Fig 4: - Topographic roughness of iron as height profile and its

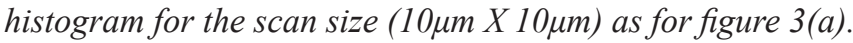

In the AFM experimental setup, the cantilever deflection is very sensitive for the measurement of the topography and the interaction between tip and sample .Cantilever deflection depends on the surface roughness of the sample ,more the surface roughness more will be the deflection and vice versa. The observation is reflected in the surface growth study employing AFM [7].

\section{Conclusion}

The surface roughness can be studied from the analysis of the height profile and topography of the etched iron by the used of AFM and hence is lead to the structure of iron. The roughness of iron obtained from the AFM is depending upon the area that is scanned. Based on the function obtained from the AFM measurements, taken on the surface areas varying on the size of the sample for which the surface roughness is agree with the height profile.

\section{References}

1) Jha, J. S. and Guglani, S.K. 1995, " The text Book Of chemistry", Surya Publication, India

2) Kittel, C. 1967, " Introduction to solid state Physics", John Wiley and Sons, India.

3) Karkare, M. 2008, " Nanotechnology-fundamentals and tools", I. K. International, New Delhi, India

4) Ramsden, J. 2009, "Nanotechnology", Ventus publishing Aps.

5) Drexler, E., Peterson, C., and Pergamit, G., 1991, " Unbounding the Future: the nanotechnology revolution", William morrow and Company, New work.

6) Lewandowski, B. R., 2009, "Scanning probe microscopy investigations of arrays of Nanoparticles". Ph. D. Thesis, Louisiana state University, New Orleans.

7) Lamichhane, S. K., 2005," MEMS: Response of a strained Silicon Semiconductor Structure" Ph. D. Thesis, JNU, India.

8) Kucharska, B. 2009," Investigation of the topography of magnetron deposited $\mathrm{Cu} / \mathrm{Ni}$ multilayers by Atomic Force Microscope", Institute of material engineering, Częstochowa University of Technology, Poland. 